\title{
Initial experience of an anal dysplasia clinic for HIV-infected men in Seattle, WA
} D Aboulafia*2,3 and L Siekas ${ }^{1}$

\begin{abstract}
Address: ${ }^{1}$ Division of Gastroenterology, Virginia Mason Medical Center, Seattle, Washington, USA, ${ }^{2}$ Division of Hematology, University of Washington, Seattle, Washington, USA and ${ }^{3}$ Division of Hematology-Oncology, Virginia Mason Medical Center, Seattle, Washington, USA

* Corresponding author
\end{abstract}

from I I th International Conference on Malignancies in AIDS and Other Acquired Immunodeficiencies (ICMAOI): Basic, Epidemiologic, and Clinical Research

Bethesda, MD, USA. 6-7 October 2008

Published: 17 June 2009

Infectious Agents and Cancer 2009, 4(Suppl 2):P38 doi:10.1186/1750-9378-4-S2-P38

This abstract is available from: http://www.infectagentscancer.com/content/4/S2/P38

(c) 2009 Aboulafia and Siekas; licensee BioMed Central Ltd.

\section{Purpose}

Anal dysplasia due to HPV infection is common in HIVinfected individuals and is a precursor to squamous cell cancer of the anus (SCCA). Herein, we describe our initial experience in assessing the incidence and severity of anal dysplasia in a newly formed anal dysplasia clinic in Seattle, WA.

\section{Methods}

We performed an IRB approved retrospective chart review of the first group of HIV seropositive individuals who underwent anal dysplasia screening with digital rectal examination, anal PAP cytology, and biopsy of disconcerting lesions. Demographic information (age, sex and race), CD4+ cell counts and HIV viral loads were recorded in conjunction with anal PAP cytology and anal biopsy results.

\section{Results}

During a seven-month start up period 150 HIV-positive men were evaluated. Their median age was 47 years (range 24 to 83 years), their median HIV viral load was $<75$ cop$\mathrm{ies} / \mathrm{mL}$ (range $<75-228,000$ copies $/ \mathrm{mL}$ ), and their median CD4+ cell counts was 454 cells/UL (range 7-1663 cells/uL). One hundred and twenty-two patients (81\%) were Caucasian, $12(8 \%)$ were African American, three (2\%) were Asian, nine (6\%) were Hispanic, and for four $(3 \%)$ race was not identified. Fifty (33\%) had a normal PAP and normal exam/biopsy, five (3\%) had an abnormal exam but either declined biopsy or had a health care condition that precluded biopsy, 48 (32\%) had low-grade anal intraepithelial neoplasia (AIN), and 47 (31\%) had high-grade AIN. One patient with high grade AIN was referred for surgical treatment and was noted to have micro-invasive SCCA. No patient experienced significant post-procedural complications (i.e. bleeding, pain, or infection). Patient tolerance and acceptance of the procedure was good and the majority of those who underwent screening have been compliant with follow up exams.

\section{Conclusion}

Anal dysplasia is common in HIV-infected men in our clinic with similar rates to those reported by other anal dysplasia clinics in urban U.S. cities. Creation of our anal dysplasia clinic has refined collaboration between HIV medicine (Internal Medicine, Hematology-Oncology, Infectious Diseases), and the departments of Gastroenterology and Surgery. The clinic is expanding rapidly with excellent community and provider support. We anticipate that the anal dysplasia clinic will allow our institution to participate in emerging HIV and HPV-related anal dysplasia clinical trials. 